\title{
Products of Message Sequence Charts ${ }^{\star}$
}

\author{
Philippe Darondeau, Blaise Genest, and Loïc Hélouët \\ IRISA, campus de Beaulieu, F-35042 Rennes Cedex
}

\begin{abstract}
An effective way to assemble partial views of a distributed system is to compute their product. Given two Message Sequence Graphs, we address the problem of computing a Message Sequence Graph that generates the product of their languages, when possible. Since all MSCs generated by a Message Sequence Graph $G$ may be run within fixed bounds on the message channels (that is, $G$ is existentially bounded), a subproblem is to decide whether the considered product is existentially bounded. We show that this question is undecidable, but turns co-NPcomplete in the restricted case where all synchronizations belong to the same process. This is the first positive result on the decision of existential boundedness. We propose sufficient conditions under which a Message Sequence Graph representing the product can be constructed.
\end{abstract}

\section{Introduction}

Scenario languages, and in particular Message Sequence Charts (MSCs) have met a considerable interest over the last decade in both academia and industry. MSCs allow for the compact description of distributed systems executions, and their visual aspect made them popular in the engineering community. Our experience with industry (France-Telecom) showed us that MSCs are most often used there together with extensions such as optional parts (that is choice) and (weak) concatenation, while iteration is left implicit. (Compositional) Message Sequence Graphs ((C)MSC-graphs) is the academic framework in which choice, weak concatenation and iteration of MSCs are formalized. For a recent survey of Message Sequence Graphs, we refer the reader to 69. A challenging problem is to automatically implement MSC-languages (that is, sets of MSCs) given by (C)MSC-graphs. Apart from the restricted case of Local Choice (C)MSC-graphs [8], this problem has received no satisfactory solution, since either deadlocks arise from the implementation [144, or implementation may exhibit unspecified behaviors 2. A further challenge is to help designing (C)MSC-graphs for complex systems, while keeping analysis and implementability decidable. Systems often result from assembling modules, reflecting different aspects. A possible way to help the modular modeling of systems into (C)MSC-graphs is thus to provide a product operator. A first attempt in this direction is 10, where the amalgamation allows the designer to merge 2 nodes of 2 MSG-graphs but not their paths. We feel that a more flexible operation, defined on MSC languages and therefore independent from MSC block decompositions, is needed.

\footnotetext{
^ Work supported by France Telecom R\&D (CRE CO2) and ANR project DOTS.
} 
Shuffling the linearizations of the languages of two (C)MSC-graphs is not the right product. On the one hand, such shuffling kills existential bounds [1], i.e., there is no upper bound on the size of the message channels within which all MSCs in the shuffled language can be run. Existential bounds are an important feature of (safe C)MSC-graphs which allow their analysis. On the other hand, two states of the (C)MSC-graphs (one for each module) may represent incompatible aspects. Hence, one needs some synchronization to control the product operation, in order to avoid incompatibilities and non existentially bounded behaviors. Control may be introduced with synchronization points: one module waits at a synchronization point until the other module reaches a compatible synchronization point, and then both can proceed. Synchronizations may be defined either per process or per state of the (C)MSC-graphs. State oriented synchronization conflicts with weak concatenation since it means that all processes of the same module pass simultaneously the synchronization barrier, which diverges strongly from the semantics of (C)MSC-graphs. Second, it harms implementability, since state-synchronized products of implementable (C)MSC-graphs may not be implementable. We therefore choose to define synchronizations per process, by means of shared local events identified by names common to both MSCs. Formally, we define thus a mixed product of MSCs that amounts to shuffling their respective events on each process, simultaneously and independently, except for the shared events that are not interleaved but coalesced. One appealing property of this definition of product is that the product of two implementable (C)MSC-graphs is also implementable (albeit with possible deadlocks), since it suffices to take the product of the implementations processwise, coalescing shared events.

In order to be represented as a (safe C)MSC-graph, an MSC language needs to be existentially bounded. So far, no algorithm is known to check the existential boundedness of an MSC language in a non-trivial case (e.g., existential boundedness is undecidable even for deterministic deadlock-free Communicating Finite State Machines, see http://perso.crans.org/ ${ }^{\sim}$ genest/GKMO\%.pdf). This is the challenging problem studied in this paper. We show that checking existential boundedness of the product of two (safe C)MSC-graphs is in general undecidable, as one expects. Surprisingly, if all shared events (synchronizations) belong to the same process, then this question becomes decidable. Once a product is known to be existentially bounded, results [124] on representative linearizations can be used. Namely, languages of MSCs defined by the globally cooperative subclass of safe CMSC-graphs have regular sets of linear representatives, where the regular representations can be computed from the CMSC-graphs and conversely. Thus, given two globally cooperative CMSC-graphs such that their product is existentially bounded, this product can be represented with a globally cooperative CMSC-graph. The authors of 4 ignore the contents of messages in the definition of MSCs. We consider messages with contents, and adapt the FIFO requirement of 4] to both weak ([213]) and strong FIFO ([1]). We recast the correspondence established in [4] into these different frameworks, and compare the complexity and decidability of these two semantics. 
The paper is organized as follows. Section 2 recalls the background of MSCs and MSC-graphs. Section 3 introduces the product of MSC-languages. Section 4 recalls the definition of existential channel bounds for MSC-languages. It is shown in Sections 5 and 6 that one can, in general, not check the existential boundedness of the product of two existentially bounded MSC-languages, whereas this problem is co-NP-complete (weak FIFO) or PSPACE (strong FIFO) when the synchronizations are attached to a single process. Section 7 defines for that special case an operation of product on CMSC-graphs. Many proofs are skipped or only briefly sketched by lack of space, but they are available in the full version of the paper, available at http://perso.crans.org/ ${ }^{\sim}$ genest/DGHOr.pdf.

\section{Background}

To begin with, we recall the usual definition of compositional Message Sequence Charts (CMSCs for short), which describe executions of communication protocols, and of CMSC-graphs, which are generators of CMSC sets. Let $\mathcal{P}, \mathcal{M}$, and $\mathcal{A}$ be fixed finite sets of processes, messages and actions, respectively. Processes may perform send events $\mathcal{S}$, receive events $\mathcal{R}$ and internal events $\mathcal{I}$. That is, the set of types of events of an MSC is $\mathcal{E}=\mathcal{S} \cup \mathcal{R} \cup \mathcal{I}$ where $\mathcal{S}=$ $\{p ! q(m) \mid p, q \in \mathcal{P}, p \neq q, m \in \mathcal{M}\}, \mathcal{R}=\{p ? q(m) \mid p, q \in \mathcal{P}, p \neq q, m \in \mathcal{M}\}$, and $\mathcal{I}=\{p(a) \mid p \in \mathcal{P}, a \in \mathcal{A}\}$. For each $p \in \mathcal{P}$, we let $\mathcal{E}_{p}=\mathcal{S}_{p} \cup \mathcal{R}_{p} \cup \mathcal{I}_{p}$ where $\mathcal{S}_{p}, \mathcal{R}_{p}$, and $\mathcal{I}_{p}$ are the restrictions of $\mathcal{S}, \mathcal{R}$, and $\mathcal{I}$, respectively, to the considered process $p\left(e . g ., \quad p ? q(m) \in \mathcal{S}_{p}\right)$. We define now MSCs over $\mathcal{E}$.

Definition 1. A compositional Message Sequence Chart $M$ is a tuple $M=$ $\left(E, \lambda, \mu,\left(<_{p}\right)_{p \in \mathcal{P}}\right)$ where

- $E$ is a finite set of events, with types $\lambda(e)$ given by a labeling map $\lambda: E \rightarrow \mathcal{E}$, - for each $p \in \mathcal{P},<_{p}$ is a total order on $E_{p}=\lambda^{-1}\left(\mathcal{E}_{p}\right)$,

$-\mu: E \rightarrow E$ is a partially defined, injective mapping,

- if $\mu\left(e_{1}\right)=e_{2}$ then $\lambda\left(e_{1}\right)=p ! q(m)$ and $\lambda\left(e_{2}\right)=q ? p(m)$ for some $p, q$ and $m$, - [weak FIFO] if $e_{1}<_{p} e_{1}^{\prime}, \lambda\left(e_{1}\right)=\lambda\left(e_{1}^{\prime}\right)=p ! q(m)$ and $\mu\left(e_{1}^{\prime}\right)$ is defined, then $\mu\left(e_{1}\right)<_{q} \mu\left(e_{1}^{\prime}\right)$ (in particular, $\mu\left(e_{1}\right)$ is defined).

- the union $<$ of $\cup_{p \in \mathcal{P}}<_{p}$ and $\cup_{e \in E}\{(e, \mu(e))\}$ is an acyclic relation.

- $M$ is an $M S C$ if the partial map $\mu$ is a bijection between $\lambda^{-1}(\mathcal{S})$ and $\lambda^{-1}(\mathcal{R})$.

Def. 1 extends the original definition of [5] (see also [4]) by considering messages with non trivial contents. There are then two alternatives to the FIFO condition. Strong FIFO requires that $e_{1}<_{p} e_{1}^{\prime}, \lambda\left(e_{1}\right)=p ! q(m), \lambda\left(e_{1}^{\prime}\right)=p ! q\left(m^{\prime}\right)$ and $\mu\left(e_{1}^{\prime}\right)$ defined entail $\mu\left(e_{1}\right)<_{q} \mu\left(e_{1}^{\prime}\right)$, i.e., there is a single channel from $p$ to $q$. The weak FIFO requirement used in Def. 1 means that there are as many FIFO channels from $p$ to $q$ as there are types of events $p ! q(m)$. In general, there are undecidable problems in the strong FIFO semantics, as weak realizability 11, which are decidable in the weak FIFO semantics [13. Anyway, all (un)decidability results established in this paper hold for both FIFO semantics, even though complexity depends on the semantics used. 
Given a CMSC $X=\left(E, \lambda, \mu,\left(<_{p}\right)_{p \in \mathcal{P}}\right)$, let $\leq_{X}$ be the reflexive and transitive closure of the relation $<$ from Def. 1] A linear extension of $X$ is an enumeration of $E$ compatible with $\leq_{X}$. A linearization of $X$ is the image of a linear extension of $X$ under the map $\lambda: E \rightarrow \mathcal{E}$ (hence it is a word of $\mathcal{E}^{*}$ ). Let $\mathcal{L}$ in $(X)$ denote the set of linearizations of $X$. For a set $\mathcal{X}$ of CMSCs, let $\mathcal{L}$ in $(\mathcal{X})$ denote the union of $\mathcal{L}$ in $(X)$ for all $X \in \mathcal{X}$. Linearizations can be defined more abstractly as follows:

Definition 2. Let $\mathcal{L}$ in $\subseteq \mathcal{E}^{*}$ be the set of all words $w$ such that for all $p, q$ and $m$, the number of occurrences $q ? p(m)$ is at most equal to the number of occurrences $p ! q(m)$ in every prefix $v$ of $w$, and both numbers are equal for $v=w$. In the strong FIFO setting, we furthermore require the equality of contents of the $i$-th emission from $p$ to $q$ and of the $i$-th reception on $q$ from $p$.

Any linearization $w$ of an $M S C$ belongs to $\mathcal{L}$ in (it may not be the case for a CMSC). Conversely, because of weak or strong FIFO, a word $w=\epsilon_{1} \ldots \epsilon_{n} \in \mathcal{L}$ in is the linearization of a unique $\operatorname{MSC}, \operatorname{Msc}(w)=\left(\{1, \ldots, n\}, \lambda, \mu,\left(<_{p}\right)\right)$, with:

$-\lambda(i)=\epsilon_{i}$ and $i<_{p} j$ if $i<j$ and $\epsilon_{i}, \epsilon_{j} \in \mathcal{E}_{p}$,

- $\mu(i)=j$ if the letter $\epsilon_{i}=p ! q(m)$ occurs $k$ times in $\epsilon_{1} \ldots \epsilon_{i}$ and the letter $\epsilon_{j}=q ? p(m)$ occurs $k$ times in $\epsilon_{1} \ldots \epsilon_{j}$ for some $p, q, m, k$.

Definition 3. Two words $w, w^{\prime} \in \mathcal{L}$ in are equivalent (notation $w \equiv w^{\prime}$ ) if $M s c(w)$ and $M s c\left(w^{\prime}\right)$ are isomorphic. For any language $\mathcal{L} \subseteq \mathcal{L}$ in, we write $[\mathcal{L}]=\left\{w \mid w \equiv w^{\prime}, w^{\prime} \in \mathcal{L}\right\}$. A language $\mathcal{L} \subseteq \mathcal{L}$ in $(\mathcal{X})$ is a representative set for a set $\mathcal{X}$ if $\mathcal{L} \cap \mathcal{L}$ in $(X) \neq \emptyset$ for all $X \in \mathcal{X}$, or equivalently, if $[\mathcal{L}]=\mathcal{L}$ in $(\mathcal{X})$.

We deduce the following properties. For any $\operatorname{MSC} X, \mathcal{L} i n(X)$ is an equivalence class in $\mathcal{L}$ in. For any $\operatorname{MSC} X$ and for any $w \in \mathcal{L}$ in, $w \in \mathcal{L}$ in $(X)$ if and only if $X$ is isomorphic to $M s c(w)$. A similar property does not hold for arbitrary CMSCs. For instance, $(p ! q(m))(q ? p(m))(q ? p(m))$ belongs to $\mathcal{L} i n(X)$ for two different CMSCs $X$, where the emission is matched by $\mu$ either with the first or with the second reception.

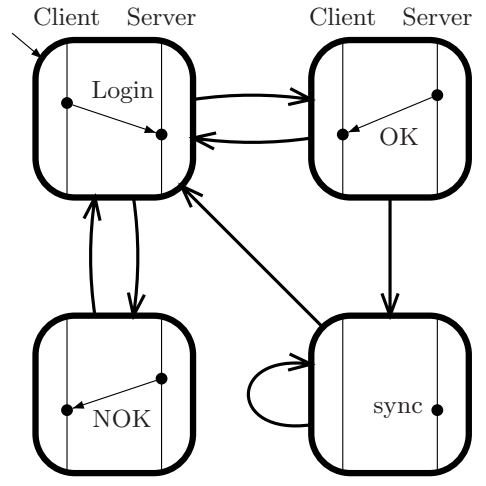

Fig. 1. Identification Scenario $G_{1}$

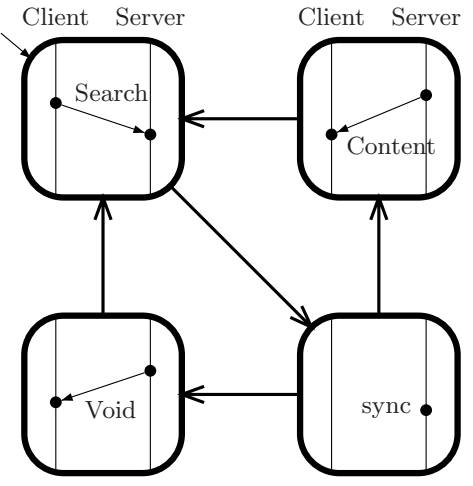

Fig. 2. Searching Scenario $G_{2}$ 
We define the concatenation $X_{1} \cdot X_{2}$ of two CMSCs $X_{i}=\left(E^{i}, \lambda^{i}, \mu^{i},\left(<_{p}^{i}\right)_{p \in \mathcal{P}}\right)$ as the set of CMSCs $X=\left(E^{1} \uplus E^{2}, \lambda^{1} \uplus \lambda^{2}, \mu,\left(<_{p}\right)_{p \in \mathcal{P}}\right)$ such that:

$-\mu \cap\left(E^{i} \times E^{i}\right)=\mu^{i}$ and $<_{p} \cap\left(E^{i} \times E^{i}\right)=<_{p}^{i}$ for $i \in\{1,2\}$ and $p \in \mathcal{P}$, $-e \in E^{2}$ and $e \leq_{X} e^{\prime}$ entail $e^{\prime} \in E^{2}$ for all $e, e^{\prime} \in E^{1} \uplus E^{2}$.

We let $\mathcal{X}_{1} \cdot \mathcal{X}_{2}$ be the union of $X_{1} \cdot X_{2}$ for all $X_{i} \in \mathcal{X}_{i}, i \in\{1,2\}$. We can now give a description of sets of MSCs with rational operations.

Definition 4. A CMSC-graph is a tuple $G=\left(V, \rightarrow, \Lambda, V^{0}, V^{f}\right)$ where $(V, \rightarrow)$ is a finite graph, $V^{0}, V^{f} \subseteq V$ are the subsets of initial and final vertices, respectively, and $\Lambda$ maps each vertex $v$ to a $C M S C \Lambda(v)$. We define $\mathcal{L}(G)$ as the set of all MSCs in $\Lambda\left(v_{0}\right) \cdot \Lambda\left(v_{1}\right) \cdot \ldots \cdot \Lambda\left(v_{n}\right)$ where $v_{0}, v_{1} \ldots, v_{n}$ is a path in $G$ from some initial vertex $v_{0} \in V^{0}$ to some final vertex $v_{n} \in V^{f}$. The $C M S C$-graph $G$ is safe if any such set $\Lambda\left(v_{0}\right) \cdot \ldots \cdot \Lambda\left(v_{n}\right)$ contains at least one $M S C$.

Intuitively, the semantics of CMSC-graphs is defined using the concatenation of the CMSCs labeling the vertices met along the paths in these graphs. Notice that $\Lambda\left(v_{0}\right) \cdot \ldots \cdot \Lambda\left(v_{n}\right)$ may contain an arbitrary number of CMSCs, but at most one of these CMSCs is an MSC. An example of a non-safe CMSC-graph is $G=\left(V, \rightarrow, \Lambda,\left\{v_{0}\right\},\left\{v_{f}\right\}\right)$ where $V=\left\{v_{0}, v_{f}\right\}, v_{0} \rightarrow v_{f}$, the $\operatorname{CMSC} \Lambda\left(v_{0}\right)$ has a single event labeled with $q ? p(m)$, and the CMSC $\Lambda\left(v_{f}\right)$ has a single event labeled with $p ! q(m)$. Indeed the two events cannot be matched by $\mu$ in $\Lambda\left(v_{0}\right) \cdot \Lambda\left(v_{f}\right)$. Notice that this is a XCMSG [12]. The reason why we do not allow XCMSGs is that safe XCMSGs are not necessarily existentially bounded, hence the Mazurkiewicz trace coding needed for the results of [4] that we use for Theorem 3 fails. Fig. 1 and 2 show two (C)MSC-graphs. Their nodes are labeled with MSCs. Concatenating $O K$ and the local event sync gives an MSC with 3 events. The reception of $O K$ and the event sync are unordered (in $G_{1}$ ). On the contrary, the event sync and the reception of Void are ordered (in $G_{2}$ ).

A safe CMSC-graph $G$ may always be expanded into a safe atomic CMSCgraph $G^{\prime}$, that is a graph in which each node is labeled with a single event, such that $\mathcal{L}(G)=\mathcal{L}\left(G^{\prime}\right)$. In the following, every safe CMSC-graph is assumed to be atomic. The expansion yields, by the way, a regular representative set for $\mathcal{L}(G)$.

\section{Product of MSC-Languages}

In order to master the complexity of distributed system descriptions, it is desirable to have at one's disposal a composition operation that allows us to weave different aspects of a system. When system aspects are CMSC-graphs with disjoint sets of processes, the concatenation of their MSC-languages can be used to this effect. Else, some parallel composition with synchronization capabilities is needed. We propose here to shuffle the events of the two MSC-graphs per process, except for the common events that serve to the synchronization. We require that all common events are internal events. Formally, what we define is an extension of the mixed product of words. The intersection with a regular 
language could be used in place of the synchronizations to control the shuffle, but this would not change significantly the results of this paper. However, synchronizing on messages could change the results, as we can encode shared events using shared messages, but not the other way around.

First, we recall the definition of the mixed product $L_{1} \| L_{2}$ of two languages $L_{1}, L_{2}$ of words (see [3]), defined on two alphabets $\Sigma_{1}, \Sigma_{2}$ not necessarily disjoint. Let $\Sigma=\Sigma_{1} \cup \Sigma_{2}$. For $i=1,2$ let $\pi_{i}: \Sigma^{*} \rightarrow \Sigma_{i}^{*}$ be the unique monoid morphism such that $\pi_{i}(\sigma)=\sigma$ for $\sigma \in \Sigma_{i}$ and $\pi_{i}(\sigma)=\varepsilon$, otherwise. Then $L_{1} \| L_{2}=\{w \mid$ $\left.\pi_{i}(w) \in L_{i}, i=\{1,2\}\right\}$ is the set of all words $w \in \Sigma^{*}$ with respective projections $\pi_{i}(w)$ in $L_{i}$; e.g., $\{a b\} \|\{c a d\}=\{c a b d, c a d b\}$ ( $a$ is the synchronizing action).

Definition 5. For $i=\{1,2\}$, let $\mathcal{X}_{i}$ be an $M S C$-language over some $\mathcal{E}^{i}$, such that $x \in \mathcal{E}^{1} \cap \mathcal{E}^{2}$ implies $x=p(a)$ for some $p$, a. The mixed product $\mathcal{X}_{1} \| \mathcal{X}_{2}$ is $\operatorname{Msc}\left(\left(\mathcal{L}\right.\right.$ in $\left(\mathcal{X}_{1}\right) \| \mathcal{L}$ in $\left.\left(\mathcal{X}_{2}\right)\right) \cap \mathcal{L}$ in $)$ and it is an $M S C$-language over $\mathcal{E}^{1} \cup \mathcal{E}^{2}$.

The mixed product operation serves to compose the languages of two CMSCgraphs that share only internal events, as is the case for the CMSC-graphs $G_{1}, G_{2}$ of Fig. 112 The synchronization sync ensures that in any MSC in $\mathcal{L}\left(G_{1}\right) \| \mathcal{L}\left(G_{2}\right)$, the server never answers a search request from the client unless the client is logged in. Thus, synchronizations serve to avoid mixing incompatible fragments of the two CMSC-graphs. When a set $\mathcal{X}$ is a singleton $\mathcal{X}=\{X\}$, we abusively write $X \| \mathcal{Y}$ instead of $\{X\} \| \mathcal{Y}$. Note that even though $X_{1}$ and $X_{2}$ are MSCs, $X_{1} \| X_{2}$ may contain more than one MSC. Under weak FIFO semantics, mixing all linearizations pairwise yields all and only linearizations of a product of MSCs. However, the product of two linearizations of strong FIFO MSCs may contain words that are not linearizations of strong FIFO MSCs. Intersecting with $\mathcal{L}$ in allows us to keep only linearizations of (strong FIFO) MSCs.

Proposition 1. $\mathcal{L}$ in $\left(\mathcal{X}_{1} \| \mathcal{X}_{2}\right)=\left(\mathcal{L}\right.$ in $\left(\mathcal{X}_{1}\right) \| \mathcal{L}$ in $\left.\left(\mathcal{X}_{2}\right)\right) \cap \mathcal{L}$ in

Lemma 1. $\left(\mathcal{L} i n\left(X_{1}\right) \| \mathcal{L} \operatorname{in}\left(X_{2}\right)\right) \cap \mathcal{L}$ in is closed under $\equiv($ see Def. 目).

However, $\left\{X_{1}\right\} \|\left\{X_{2}\right\}$ may be larger than $M s c\left(w_{1} \| w_{2}\right)$ for fixed representations $w_{1} \in \mathcal{L}$ in $\left(X_{1}\right)$ and $w_{2} \in \mathcal{L} i n\left(X_{2}\right)$. This situation is illustrated with

$$
\begin{aligned}
& w_{1}=\left(p ! q\left(m_{1}\right)\right)\left(q ? p\left(m_{1}\right)\right)\left(p ! q\left(m_{1}\right)\right)\left(q ? p\left(m_{1}\right)\right), \\
& w_{1}^{\prime}=\left(p ! q\left(m_{1}\right)\right)^{2}\left(q ? p\left(m_{1}\right)\right)^{2}, \\
& w_{2}=\left(q ! p\left(m_{2}\right)\right)\left(p ? q\left(m_{2}\right)\right)\left(q ! p\left(m_{2}\right)\right)\left(p ? q\left(m_{2}\right)\right), \\
& w_{2}^{\prime}=\left(q ! p\left(m_{2}\right)\right)^{2}\left(p ? q\left(m_{2}\right)\right)^{2}, \\
& w_{3}=\left(p ! q\left(m_{1}\right)\right)^{2}\left(q ! p\left(m_{2}\right)\right)^{2}\left(p ? q\left(m_{2}\right)\right)^{2}\left(q ? p\left(m_{1}\right)\right)^{2} .
\end{aligned}
$$

and $X_{1}=M s c\left(w_{1}\right)=M s c\left(w_{1}^{\prime}\right), X_{2}=M s c\left(w_{2}\right)=M s c\left(w_{2}^{\prime}\right), X_{3}=M s c\left(w_{3}\right)$. There is no synchronization. Now $X_{3} \in M s c\left(w_{1}^{\prime} \| w_{2}^{\prime}\right)$, but $X_{3} \notin M s c\left(w_{1} \| w_{2}\right)$. This observation shows that products must be handled with care. Indeed, an advantage of CMSC-graphs is to represent large sets of linearizations with small subsets of representatives. However, $w_{1}$ is a representative for $X_{1}, w_{2}$ is for $X_{2}$, but $w_{1} \| w_{2}$ is not a set of representatives for $X_{1} \| X_{2}$. 


\section{Bounds for MSCs and Products}

We review in this section ways of classifying CMSC-graphs based on bounds for communication channels, and we examine how these bounds behave under product of CMSC-languages. We focus on MSC-languages with regular representative sets. As indicated earlier, a regular representative set for the language of a safe CMSC-graph $G$ may be obtained by expanding $G$ into an atomic CMSCgraph $G^{\prime}$. As observed in [12, it follows from a pumping lemma that whenever $\mathcal{L} \subseteq \mathcal{L}$ in is a regular representative set for some $\mathcal{X}$, the words in $\mathcal{L}$ are uniformly $B$-bounded, for some $B>0$, as defined hereafter. First, the definition of a channel depends on the semantics. In the weak FIFO setting, a channel is a triple $p, q \in \mathcal{P}, m \in \mathcal{M}$, and $p ! q(m)$ is an emission $(q ? p(m)$ is a reception) on this channel. In the strong FIFO setting, a channel is a pair $p, q \in \mathcal{P}$, and $p ! q(m)$ is an emission $\left(q ? p\left(m^{\prime}\right)\right.$ is a reception) on this channel for any $m, m^{\prime} \in \mathcal{M}$. A word $w \in \mathcal{E}^{*}$ is $B$-bounded if, for any prefix $v$ of $w$ and any channel $c$, the number of emissions on $c$ in $v$ exceeds the number of receptions on $c$ in $v$ by at most $B$.

A MSC $X$ is $\forall$-B-bounded if every linearization $w \in \mathcal{L}$ in $(X)$ is $B$-bounded. A MSC $X$ is $\exists$-B-bounded if some linearization $w \in \mathcal{L}$ in $(X)$ is $B$-bounded. A set of MSCs $\mathcal{X}$ is $\exists$ - $B$-bounded if all MSCs $X \in \mathcal{X}$ are $\exists$ - $B$-bounded; $\mathcal{X}$ is existentially bounded if it is $\exists$-B-bounded for some $B$. Let $\mathcal{L}_{i n}^{B}(\mathcal{X})$ denote the set of $B$ bounded words $w$ in $\mathcal{L}$ in $(\mathcal{X})$. Clearly, any $\mathcal{X}$ with a regular representative set is existentially $B$-bounded for some $B$, but it may not be $\forall$ - $B$-bounded for any $B$. Conversely, when an MSC-language $\mathcal{X}$ is $\exists$ - $B$-bounded, $\operatorname{Lin}^{B}(\mathcal{X})$ is a representative set for $\mathcal{X}$, but it is not necessarily a regular language.

Proposition 2. $\mathcal{L}_{i n}^{B}\left(\mathcal{X}_{1} \| \mathcal{X}_{2}\right)=\left(\mathcal{L}_{i n}{ }^{B}\left(\mathcal{X}_{1}\right) \| \mathcal{L} i n^{B}\left(\mathcal{X}_{2}\right)\right) \cap \mathcal{L}_{i n}^{B}$.

The above result shows that the mixed product behaves nicely with respect to bounded linearizations. If $\mathcal{X}_{1}$ and $\mathcal{X}_{2}$ are $\forall$-B-bounded, then $\operatorname{Lin}\left(\mathcal{X}_{i}\right)=$ $\mathcal{L}_{i n}{ }^{B}\left(\mathcal{X}_{i}\right)$, and using Prop. [1] their product is also $\forall-B$-bounded. However, it may occur that both $\mathcal{X}_{1}$ and $\mathcal{X}_{2}$ are $\exists$ - $B$-bounded but their mixed product is not existentially bounded. For instance, for all $j$, let $X_{1}^{j}$ be the MSC with $j$ messages $m_{1}$ from $p$ to $q$ and $X_{2}^{j}$ be the MSC with $j$ messages $m_{2}$ from $q$ to $p$. All these MSCs are $\exists$-1-bounded since $\left(p ! q\left(m_{1}\right) q ? p\left(m_{1}\right)\right)^{j} \in \mathcal{L}$ in $\left(X_{1}^{j}\right)$ is 1-bounded. Define $\mathcal{X}_{1}=\left\{X_{1}^{j} \mid j>0\right\}$ and $\mathcal{X}_{2}=\left\{X_{2}^{j} \mid j>0\right\}$, thus $\mathcal{X}_{1}, \mathcal{X}_{2}$ are $\exists$-1-bounded, but $\mathcal{X}_{1} \| \mathcal{X}_{2}$ is not $\exists$ - $B$-bounded for any $B$ since $M s c\left(p ! q\left(m_{1}\right)^{B}\left(q ! p\left(m_{2}\right) p ? q\left(m_{2}\right)\right)^{B} q ? p\left(m_{1}\right)^{B}\right) \in \mathcal{X}_{1} \| \mathcal{X}_{2}$, but it is not $\exists-(B-1)$ bounded.

Definition 6. Given an $M S C X=\left(E, \lambda, \mu,\left(<_{p}\right)_{p \in \mathcal{P}}\right)$ and a non-negative integer $B$, let $\operatorname{Rev}_{B}$ be the binary relation on $E$ such that Rev $_{B} e^{\prime}$ if and only if, for some channel $c$, $e$ is the $i$-th reception on channel $c$ and $e^{\prime}$ is the $i+B$-th emission on channel c. We also define Rev $v_{\geq B}=\cup_{B^{\prime} \geq B} \operatorname{Rev}_{B^{\prime}}$.

Proposition 3 (lemma 2 in [11]). A MSC $X$ is $\exists$-B-bounded if and only if the relation $<\cup R e v_{B}$ is acyclic, if and only if the relation $<\cup R e v_{\geq B}$ is acyclic. 
If $X$ is $\exists$ - $B$-bounded then $X$ is $\exists$ - $B^{\prime}$-bounded for all $B^{\prime} \geq B$, because $R e v_{B^{\prime}}$ is included in the least order relation containing $\operatorname{Rev}_{B}$ and $\bigcup_{p \in \mathcal{P}}<_{p}$. For instance, in $M s c\left(p ! q\left(m_{1}\right)^{B}\left(q ! p\left(m_{2}\right) p ? q\left(m_{2}\right)\right)^{B} q ? p\left(m_{1}\right)^{B}\right)$ let $\left(a_{i}, b_{i}\right)$ denote the $i$ th pair of events $\left(p ! q\left(m_{1}\right), q ? p\left(m_{1}\right)\right)$ and $\left(c_{i}, d_{i}\right)$ the $i$-th pair of events $\left(q ! p\left(m_{2}\right)\right.$, $\left.p ? q\left(m_{2}\right)\right)$, then $a_{B}<_{p} d_{1} \operatorname{Rev}_{(B-1)} c_{B}<_{q} b_{1} \operatorname{Rev}_{(B-1)} a_{B}$ is a cycle.

\section{Monitored Product of MSC-Languages}

It is important to analyze formally MSC-languages, since following paths in MSC-graphs does not help grasping all the generated scenarios. Most often, in decidable cases 7 [16, the analysis of an MSC-language $\mathcal{X}$ amounts to check either the membership of a given MSC $X$, or whether $\mathcal{L}$ in $(\mathcal{X})$ has an empty intersection with a regular language $L$ (representing the complement of a desired property). In the case of a product language $\mathcal{X}_{1} \| \mathcal{X}_{2}$, membership can be checked using the projections, since $X \in \mathcal{X}_{1} \| \mathcal{X}_{2}$ if and only if $\pi_{i}(X) \in \mathcal{X}_{i}$ for $i=1,2$. However, in order to analyse regular properties of $\mathcal{L}\left(G_{1}\right) \| \mathcal{L}\left(G_{2}\right)$, one often needs computing a safe CMSC-graph $G$ such that $\mathcal{L}(G)=\mathcal{L}\left(G_{1}\right) \| \mathcal{L}\left(G_{2}\right)$. In particular, one needs an existential bound $B$ for the product. Unfortunately, the theorem below shows that one cannot decide whether such $G$ exists when $G_{1}$ and $G_{2}$ share events on two processes or more.

Theorem 1. Let $G_{1}, G_{2}$ be two (safe C)MSC-graphs. It is undecidable whether $\mathcal{L}\left(G_{1}\right) \| \mathcal{L}\left(G_{2}\right)$ is existentially bounded, in both weak and strong FIFO semantics.

Proof. We show that the Post correspondence problem may be reduced to the above decision problem. Given two finite lists of words $u_{1}, \ldots, u_{n}$ and $w_{1}, \ldots, w_{n}$ on some alphabet $\Sigma$ with at least two symbols, the problem is to decide whether $u_{i_{1}} u_{i_{2}} \ldots u_{i_{k}}=w_{i_{1}} w_{i_{2}} \ldots w_{i_{k}}$ for some non-empty sequence of indices $i_{1} \cdots i_{k}$. This problem is known to be undecidable for $n>7$. Given an instance of the Post correspondence problem, i.e., two lists of words $u_{1}, \ldots, u_{n}$ and $w_{1}, \ldots, w_{n}$ on $\Sigma$, consider the two MSC-graphs $G_{1}=\left(V, \rightarrow, \Lambda_{1}, V^{0}, V^{f}\right)$ and $G_{2}=(V, \rightarrow$, $\left.\Lambda_{2}, V^{0}, V^{f}\right)$, with the same underlying graph $\left(V, \rightarrow, V^{0}, V^{f}\right)$, constructed as follows $\left(G_{1}\right.$ is partially shown in Fig. 3).

Define $V=\left\{v_{0}, v_{1}, \ldots, v_{n}, v_{n+1}\right\}$ with $V^{0}=\left\{v_{0}\right\}$ and $V^{f}=\left\{v_{n+1}\right\}$. Let $v_{0} \rightarrow v_{i}, v_{i} \rightarrow v_{j}$, and $v_{i} \rightarrow v_{n+1}$ for all $i, j \in\{1, \ldots, n\}$ (where possibly $i=j$ ). Finally let $v_{n+1} \rightarrow v_{n+1}$.

For each $v \in V, \Lambda_{1}(v)$ is a finite MSC over $\mathcal{P}_{1}=\{p, q\}, \mathcal{A}_{1}=\{1, \ldots, n\} \cup \Sigma$, $\mathcal{M}_{1}=\left\{m_{1}, m_{1}^{\prime}\right\}$. Actions $i \in\{1, \ldots, n\}$ represent indices of pairs of words $\left(u_{i}, v_{i}\right)$ and they occur on process $p$. Actions $\sigma \in \Sigma$ represent letters of words $u_{i}$ and they occur on process $q$. Let $\Lambda_{1}\left(v_{0}\right)$ be the empty MSC. For $i \in\{1, \ldots, n\}$, let $\Lambda_{1}\left(v_{i}\right)$ be the MSC with $p ! q\left(m_{1}\right)$ followed by $p(i)$ on process $p$ and with $q$ ? $p\left(m_{1}\right)$ followed by the sequence $q\left(\sigma_{i, 1}\right) q\left(\sigma_{i, 2}\right) \ldots q\left(\sigma_{i, l_{i}}\right)$, representing $u_{i}=\sigma_{i, 1} \sigma_{i, 2} \ldots \sigma_{i, l_{i}}$, on process $q$. Finally let $\Lambda_{1}\left(v_{n+1}\right)$ be the MSC with the events $p ! q\left(m_{1}^{\prime}\right)$ and $q ? p\left(m_{1}^{\prime}\right)$ on processes $p$ and $q$, respectively.

For each $v \in V, \Lambda_{2}(v)$ is a finite MSC over $\mathcal{P}_{2}=\{p, r, q\}, \mathcal{A}_{2}=\{1, \ldots, n\} \cup \Sigma$, $\mathcal{M}_{2}=\left\{m_{2}, m_{2}^{\prime \prime}, m_{2}^{\prime}\right\}$. For $i=0, \ldots, n, \Lambda_{2}\left(v_{i}\right)$ is defined alike $\Lambda_{1}\left(v_{i}\right)$ but now 


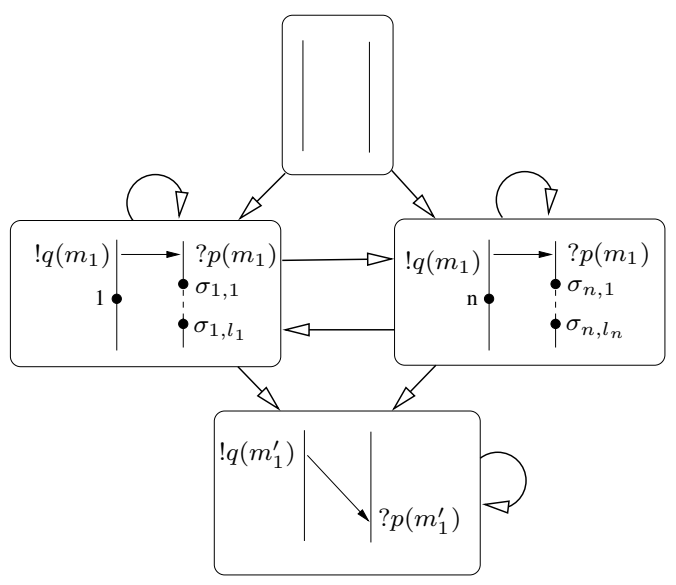

Fig. 3.

replacing the message $p ! q\left(m_{1}\right), q ? p\left(m_{1}\right)$ with two messages $p ! r\left(m_{2}\right), r ? p\left(m_{2}\right)$, $r ! q\left(m_{2}^{\prime \prime}\right), q ? r\left(m_{2}^{\prime \prime}\right)$ and $u_{i}$ with $w_{i} \cdot \Lambda_{2}\left(v_{n+1}\right)$ is the MSC with the events $p ? q\left(m_{2}^{\prime}\right)$ and $q ! p\left(m_{2}^{\prime}\right)$ on processes $p$ and $q$, respectively.

For $i=1,2$ let $\mathcal{X}_{i}=\mathcal{L}\left(G_{i}\right)$, then $\mathcal{L}_{i n}^{1}\left(\mathcal{X}_{i}\right)$ is a regular representative set for $\mathcal{X}_{i}$. If the Post correspondence problem has no solution, then $\mathcal{X}_{1} \| \mathcal{X}_{2}$ is empty, hence it is existentially bounded. In the converse case, $\mathcal{X}_{1} \| \mathcal{X}_{2}$ contains for all $B$ some MSC including a crossing of $B$ messages $m_{1}^{\prime}$ by $B$ messages $m_{2}^{\prime}$, hence it is not existentially bounded.

The proof of Theorem 1 is inspired by the proof that $\mathcal{L}\left(G_{1}\right) \cap \mathcal{L}\left(G_{2}\right)=\emptyset$ is undecidable for generic MSC-graphs $G_{1}, G_{2}$ [15]. Theorem 1 motivates the introduction of a monitor process $m p$ and a monitored product in which all synchronizations are (internal) events located on the monitor process. The set of synchronizations is denoted by $\mathcal{S E}$. The monitored product $\mathcal{X}_{1} \|_{\mathrm{mp}} \mathcal{X}_{2}$ of sets $\mathcal{X}_{1}$ and $\mathcal{X}_{2}$ on monitor process $m p \in \mathcal{P}$ is defined only if $\mathcal{S E} \subseteq\{m p(a) \mid a \in \mathcal{A}\}$. In that case, we set $\mathcal{X}_{1}\left\|_{\mathrm{mp}} \mathcal{X}_{2}=\mathcal{X}_{1}\right\| \mathcal{X}_{2}$. For instance, in the monitored product $\mathcal{L}\left(G_{1}\right) \|_{\mathrm{mp}} \mathcal{L}\left(G_{2}\right)$ of the CMSC-graphs of Fig. 1 and Fig. 2, we can choose $m p=$ server and $\mathcal{S E}=\{m p($ sync $)\}$. The adequacy of the monitored product to weave aspects of a distributed system is confirmed by the following theorem, which holds for both strong and weak FIFO semantics. We conjecture that the problem is PSPACE-complete in the strong FIFO case.

Theorem 2. Given two safe CMSC-graphs $G_{1}, G_{2}$, one can decide whether the monitored product of $\mathcal{L}\left(G_{1}\right)$ and $\mathcal{L}\left(G_{2}\right)$ is $\exists$-bounded. The problem is co-NPcomplete and in PSPACE for weak and strong FIFO semantics respectively.

The next section sketches a proof for this theorem. Notice that the proof is trivial in the case where $G_{1}, G_{2}$ have disjoint sets of processes except for $m p$. Then, $\mathcal{L}\left(G_{1}\right) \|_{\mathrm{mp}} \mathcal{L}\left(G_{2}\right)$ is existentially bounded (with the bound given by the maximum of the minimal existential bounds of $\mathcal{L}\left(G_{1}\right)$ and $\left.\mathcal{L}\left(G_{2}\right)\right)$. 


\section{Checking Existential Boundedness}

We prove Theorem 2 in two stages. First, we show that if the monitored product $\mathcal{L}\left(G_{1}\right) \|_{\mathrm{mp}} \mathcal{L}\left(G_{2}\right)$ is existentially bounded, then this property holds for a 'small' bound with respect to the size of $G_{1}$ and $G_{2}$.

Proposition 4. Given two safe $C M S C$-graphs $G_{1}$ and $G_{2}$, the $M S C$-language $\mathcal{L}\left(G_{1}\right) \|_{\mathrm{mp}} \mathcal{L}\left(G_{2}\right)$ is existentially bounded if and only if it is existentially $B^{w}$ bounded (resp. $B^{s}$-bounded) for weak (resp. strong) FIFO semantics, where $B^{w}=$ $2 K_{1} B^{\prime}, B^{s}=2 K_{2} K_{3} B^{\prime}$, and $K_{1}, K_{2}, B^{\prime}$ (resp. $K_{3}$ ) are polynomial (resp. exponential) in the size of $P, G_{1}, G_{2}$.

Then we show that one can check whether the monitored product of $\mathcal{L}\left(G_{1}\right)$ and $\mathcal{L}\left(G_{2}\right)$ is $\exists$ - $B$-bounded, using the bounds $B^{w}, B^{s}$ of Prop. 4. Notice that $B^{s}$ written in binary is of size polynomial in $\left|G_{1}\right|+\left|G_{2}\right|$.

Proposition 5. Given two safe $C M S C$-graphs $G_{1}, G_{2}$ and an integer $B$, it is co-NP-complete (resp. PSPACE) to decide whether $\mathcal{L}\left(G_{1}\right) \|_{\mathrm{mp}} \mathcal{L}\left(G_{2}\right)$ is $\exists$-Bbounded, for weak (resp. strong) FIFO semantics. The PSPACE result holds also when $B$ is written in binary.

\section{Graph representation of monitored products}
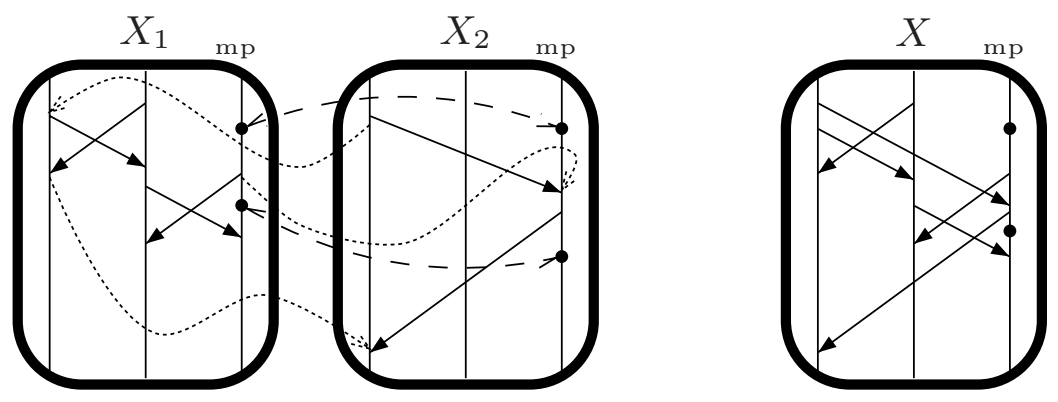

Fig. 4. $X \in X^{1} \|_{\mathrm{mp}} X^{2}$ and the corresponding relations $\rightarrow^{1} \cup \rightarrow^{2}, \leftrightarrow$

These two results are obtained using special representations for MSCs constructed by monitored product. Let $X \in X_{1} \|_{\mathrm{mp}} X_{2}$ then $\exists w \in \mathcal{L}$ in: $X=$ $\operatorname{Msc}(w)$ and $\pi_{i}(w)=w_{i} \in \mathcal{L} i n\left(X_{i}\right)$. The MSC $X$ is determined up to isomorphism by its projections on processes, because of FIFO. More precisely, for each $p \in \mathcal{P}, \pi_{p}(w) \in \pi_{p}\left(w_{1}\right) \| \pi_{p}\left(w_{2}\right)$. Moreover, for $p=m p, \pi_{p}\left(w_{1}\right)$ and $\pi_{p}\left(w_{2}\right)$ have the same projection on $\mathcal{S E}$. Therefore the projection $\left(E_{p},<_{p}\right)$ of $X$ on each process $p$ may be seen as an interleaving of $\left(E_{p}^{1},<_{p}^{1}\right)$ and $\left(E_{p}^{2},<_{p}^{2}\right)$ where the synchronized pairs of events $e_{1} \in E_{m p}^{1}$ and $e_{2} \in E_{m p}^{2}$ with labels in $\mathcal{S E}$ are coalesced. Let $\longleftrightarrow \subseteq E_{m p}^{1} \times E_{m p}^{2}$ be the relation comprising synchronized pairs of events. For each $p \in \mathcal{P}$, let $\rightarrow{ }_{p}^{1} \subseteq E_{p}^{2} \times E_{p}^{1}$ (resp. $\rightarrow_{p}^{2} \subseteq E_{p}^{1} \times E_{p}^{2}$ ) be the relation comprising ordered pairs of events $e_{2} e_{1}$ (resp. $e_{1} e_{2}$ ) switching from $E_{p}^{2}$ to $E_{p}^{1}$ 
(resp. $E_{p}^{1}$ to $\left.E_{p}^{2}\right)$ in the interleaved sequence $\left(E_{p},<_{p}\right)$. The MSC $X$ may now be represented by the juxtaposition of $X_{1}$ and $X_{2}$ interlinked with $\longleftrightarrow$ and with the relations $\rightarrow_{p}^{1}$ and $\rightarrow_{p}^{2}$ for all $p \in \mathcal{P}$. The result is a graph, that we denote $X_{1 \|_{\mathrm{mp}} 2}$, with set of nodes $E^{1} \cup E^{2}$. Conversely, any acyclic graph connecting $X_{1}$ and $X_{2}$ with relations $\rightarrow_{p}^{i}$ and $\longleftrightarrow$ represents a non-empty set of weak FIFO MSCs $\mathcal{X}$. We say that the transitive closure $<_{\|_{\mathrm{mp}}}$ of $<_{i}^{p}, \rightarrow_{p}^{i}$ and $\longleftrightarrow$ is compatible with strong FIFO if there do not exist two messages $(s, r),\left(s^{\prime}, r^{\prime}\right)$ on the same channel $c$ such that $s<s^{\prime}$ and $r^{\prime}<r$. There may be several such MSCs if for some $p$ the relation $\rightarrow_{p}^{1} \cup \rightarrow_{p}^{2} \cup<_{p}^{1} \cup<_{p}^{2}$ is not a total order on $E_{p}$. Otherwise, the original MSC $X$ may be reconstructed from $X_{1 \|_{\mathrm{mp}} 2}$ as follows: $E$ is the quotient of $E^{1} \cup E^{2}$ by the equivalence relation $\longleftrightarrow$ and $<=<\|\left._{\mathrm{mp}}\right|_{E}$. For an illustration, see Fig. 4 where the edges of the graph represent the relations $<_{p}^{i}, \mu_{i}, \longleftrightarrow$ (dashed) and $\rightarrow_{p}^{i}$ (dotted). The graph is compatible with strong FIFO. A unique MSC $X$ can be reconstructed from it, depicted on the right of the figure. More formally, we can state the following lemma:

Lemma 2. Let $G_{1}$ and $G_{2}$ be safe and atomic $C M S C$-graphs and $B$ an integer. Then $\mathcal{L}\left(G_{1}\right) \|_{\mathrm{mp}} \mathcal{L}\left(G_{2}\right)$ is $\exists$-B-bounded if and only if, for any synchronized pair of $M S C s X_{1} \in \mathcal{L}\left(G_{1}\right)$ and $X_{2} \in \mathcal{L}\left(G_{2}\right)$ with respective sets of events $E^{1}$ and $E^{2}$, there is no subset $\left\{e_{1}, \ldots, e_{n}\right\} \subseteq E^{1} \cup E^{2}$ with at most two events in $E_{p}^{1} \cup E_{p}^{2}$ for each process $p \in \mathcal{P}$ such that:

1. for all $j,\left(e_{j}, e_{(j+1)} \bmod n\right)$ belongs to one of the relations $<^{i}$, Rev $v_{B}$, or $E_{p}^{i} \times E_{p}^{3-i}$ for $i=1$ or 2 and $p \in \mathcal{P}$,

2. there is no proper cycle in $\left\{e_{1}, \ldots, e_{n}\right\}$ w.r.t. the transitive closure $<_{\|_{\mathrm{mp}}}$ of the relation $<^{1} \cup<^{2} \cup \longleftrightarrow \cup \rightarrow$ where $\longleftrightarrow$ is the synchronizing relation among coalesced events, and $e \rightarrow e^{\prime}$ if $e=e_{j} \in E_{p}^{i}$ and $e^{\prime}=e_{(j+1)} \bmod n \in$ $E_{p}^{3-i}$ for some $j \in\{1, \ldots n\}, i \in\{1,2\}$ and $p \in \mathcal{P}$,

3. in the strong FIFO case, $<_{\|_{\mathrm{mp}}}$ is compatible with strong FIFO.

The proofs of Prop. 4 and 5 are based on synchronized paths and Lemma 3 . A synchronized path $\alpha \beta_{1} \cdots \beta_{n} \gamma$ of $G_{1}, G_{2}$ is a sequence of pairs of paths $\alpha=$ $\left(\alpha^{1}, \alpha^{2}\right), \beta_{i}=\left(\beta_{i}^{1}, \beta_{i}^{2}\right), \gamma=\left(\gamma^{1}, \gamma^{2}\right)$, where $\alpha^{k} \beta_{1}^{k} \cdots \beta_{n}^{k} \gamma^{k}$ is a path of $G_{k}, \pi_{\mathcal{S E}}\left(\alpha^{1}\right)$ $=\pi_{\mathcal{S E}}\left(\alpha^{2}\right), \pi_{\mathcal{S E}}\left(\beta_{i}^{1}\right)=\pi_{\mathcal{S E}}\left(\beta_{i}^{2}\right)$ and $\pi_{\mathcal{S E}}\left(\gamma^{1}\right)=\pi_{\mathcal{S E}}\left(\gamma^{2}\right)$. Furthermore, $\beta_{i}^{k}$ is a loop of $G_{k}$ for all $i, k$. Lemma 3 claims that if $n$ is sufficiently large, there exists a synchronized loop of $\rho_{2}$ which has no contribution to the ordering between events in $\rho_{1}$ and $\rho_{2}$. This loop can thus be removed or iterated without compromising acyclicity, and is compatible with strong FIFO if needed.

Lemma 3. Let $G_{1}$ and $G_{2}$ be safe and atomic $C M S C$-graphs, $K$ be an integer and $\left(\alpha^{1}, \alpha^{2}\right)\left(\beta_{1}^{1}, \beta_{1}^{2}\right) \cdots\left(\beta_{K}^{1}, \beta_{K}^{2}\right)\left(\gamma^{1}, \gamma^{2}\right)$ be a synchronized path of $G_{1}, G_{2}$. Let $\rightarrow$ be a partial order on a set $E$ of $n \leq 2|\mathcal{P}|$ events of $\alpha^{1} \cup \alpha^{2} \cup \gamma^{1} \cup \gamma^{2}$ compatible with the order of the synchronized path. For all $j \geq i \geq 1, \ell \geq 0$, we denote by $<_{i, j}^{\ell}$ the relation on $\left(\alpha^{1}, \alpha^{2}\right)\left(\beta_{1}^{1}, \beta_{1}^{2}\right) \cdots\left[\left(\beta_{i}^{1}, \beta_{i}^{2}\right) \cdots\left(\beta_{j}^{1}, \beta_{j}^{2}\right)\right]^{\ell} \cdots\left(\beta_{K}^{1}, \beta_{K}^{2}\right)\left(\gamma^{1}, \gamma^{2}\right)$ generated by the synchronizations and the relation $\rightarrow$. 
- For all $i, j, i^{\prime}, j^{\prime},<_{i, j}^{1}=<_{i^{\prime}, j^{\prime}}^{1}$, denoted $<$, and this relation is a partial order.

- Let $K_{1}, K_{2}, K_{3}$ be the constants of Prop. 4.

- If $K>K_{1}$, then there exists $i$ such that for all $x, y \in \alpha^{1} \cup \alpha^{2} \cup \gamma^{1} \cup \gamma^{2}$ and $l \geq 0$, we have $x<_{i, i}^{\ell} y$ iff $x<y$ (in particular, $<_{i, i}^{\ell}$ is a partial order).

- If $K>K_{2} K_{3}$ and $<$ is compatible with strong FIFO, then there exist $i, j$ such that $<_{i, j}^{\ell}$ is an order compatible with strong FIFO, for all $l \geq 0$.

\section{$\star$ General outline of the proof for Prop. 4}

Let $X \in \mathcal{L}\left(G_{1}\right) \|_{\mathrm{mp}} \mathcal{L}\left(G_{2}\right)$, thus $X$ may be represented in product form by $X_{1 \|_{\mathrm{mp}} 2}=\left(X_{1}, X_{2}, \longleftrightarrow,\left(\rightarrow_{p}^{i}\right)_{p \in \mathcal{P}}^{i=1,2}\right)$. Suppose that $X$ is not $\exists$ - $B$-bounded for some $B=2 K B^{\prime}$. By Prop. 3 , $<\cup R e v_{\geq B}$ has a cycle in $X$. We have $\operatorname{Rev}_{\geq B}^{1} \cup$ $R e v_{\geq B}^{2} \subseteq R e v_{\geq B} \subseteq R e v_{\geq B / 2}^{1} \cup R e v_{\geq B / 2}^{2}$. Therefore, the union of $\longleftrightarrow$ and the relations $<^{i}, R e v_{\geq K B^{\prime}}^{i}$, and $\rightarrow_{p}^{i}$ for $i=1,2$ has a cycle $e_{1} e_{2} \ldots e_{m}$ with $e_{j} \neq e_{k}$ for $j \neq k$. We let $e_{m+1}=e_{1}$. One can assume that $e_{1} e_{2} \ldots e_{m}$ contains no synchronization event with shared label and at most two events on each process $p$ (Lemma 5.5 in [4]), hence $m \leq 2|\mathcal{P}|$. Furthermore, there is at least one pair of events $\left(e_{j}, e_{j+1}\right)$ in $\operatorname{Rev}_{B_{j}}^{i}$, w.l.o.g. $e_{1} \operatorname{Rev}_{B_{1}}^{1} e_{2}$, with $B_{1} \geq K B^{\prime}$. Notice that $\left(e_{1} \cdots e_{m}\right)$ is also a cycle for the union of $\longleftrightarrow,<^{i}, \operatorname{Rev}_{\geq K B^{\prime}}^{i}$, and $\rightarrow{ }_{p}^{i} \cap\left(e_{j}, e_{j+1}\right)_{j \leq m}$ for $i=1,2$, that is we need to consider only a linear number of pairs in $\rightarrow_{p}^{i}$. We construct MSCs $X_{1}^{\prime} \in \mathcal{L}\left(G_{1}\right), X_{2}^{\prime} \in \mathcal{L}\left(G_{2}\right)$ embedding $X_{1}, X_{2}$ via $\phi: X_{i} \hookrightarrow X_{i}^{\prime}$ such that $\phi\left(e_{1}\right) \phi\left(e_{2}\right) \ldots \phi\left(e_{m}\right)$ is a cycle for $\phi\left(R e v \geq K B^{\prime}\right) \cup<_{X^{\prime}}$, where $\left(X^{\prime},<_{X^{\prime}}\right)$ is the oriented graph obtained by connecting $X_{1}^{\prime}$ and $X_{2}^{\prime}$ with $\longleftrightarrow$ and $\phi\left(\rightarrow \begin{array}{l}i \\ p\end{array}\right) \cap\left(\phi\left(e_{j}\right), \phi\left(e_{j+1}\right)\right)_{j \leq m}$. More precisely, $X_{1}^{\prime}, X_{2}^{\prime}$ are such that $\phi\left(e_{1}\right) \operatorname{Rev}_{\geq 2 B_{1}+1}^{1} \phi\left(e_{2}\right)$ and $e_{j} \operatorname{Rev}_{B_{j}}^{i} e_{j+1} \Rightarrow \phi\left(e_{j}\right) \operatorname{Rev}_{\geq B_{j}}^{i} \phi\left(e_{j+1}\right)$ for $j \neq 1$ and $B_{j} \geq K B^{\prime}$. As soon as $<_{X^{\prime}}$ is a partial order (compatible with strong FIFO if needed), Prop. 4 follows by induction and by applying Lemma 2.

\section{* General outline of the proof for Prop. 5}

In order to conclude that $\mathcal{L}\left(G_{1}\right) \|_{\mathrm{mp}} \mathcal{L}\left(G_{2}\right)$ is not $\exists$ - $B$-bounded, one should search for MSCs $X_{1} \in \mathcal{L}\left(G_{1}\right), X_{2} \in \mathcal{L}\left(G_{2}\right)$, and $X \in\left(X_{1} \|_{\mathrm{mp}} X_{2}\right)$ such that $<_{X} \cup \operatorname{Rev}_{B}$ contains a cycle. In the weak FIFO setting, we use a small model property. Assume that the product of $\mathcal{L}\left(G_{1}\right)$ and $\mathcal{L}\left(G_{2}\right)$ is not existentially $B^{w}$ bounded. We apply Lemma 2 to obtain a synchronized pair of paths $\rho_{1}, \rho_{2}$ of $G_{1}, G_{2}$, with a set $E$ of at most $2|\mathcal{P}|$ events, and a relation $\rightarrow \in E \times E$ which creates a cycle with $<^{i} \cup R e v_{B^{w}}^{i}$. By contradiction, assume that the minimal size of such a synchronized path $\left(\rho_{1}, \rho_{2}\right)$ (that is its number of transitions) is larger than $\left(\left(4|\mathcal{P}| B^{w}+1\right) K_{1} B^{\prime}\right.$, then it contains $\left(4|\mathcal{P}| B^{w}+1\right) K_{1}$ synchronized pairs of loops. Applying Lemma 3 with $\ell=0$, we know that there are $4|\mathcal{P}| B^{w}+1$ loops which can be individually deleted without changing the order on $E$. There are at most $2|\mathcal{P}| B^{w}$ messages which can affect the $\operatorname{Rev} v_{B^{w}}^{i}$ relation, hence $4|\mathcal{P}| B^{w}$ loops which contain some emission or reception of such messages. Therefore, one synchronized pair of loops can be deleted without changing the order on $E$ nor the $\operatorname{Rev}_{B^{w}}^{i}$ relations, which contradicts the minimality of $\rho_{1}, \rho_{2}$. To obtain 
a co-NP algorithm, it suffices to guess a path of $G_{1}$ and a path of $G_{2}$ of size polynomial, to guess $2|\mathcal{P}|$ events, and to check in polynomial time that there is no cycle in $<^{1} \cup<^{2} \cup \leftrightarrow \cup \rightarrow$, whereas there is a cycle in $<^{1} \cup<^{2} \cup \leftrightarrow \cup \rightarrow$ $\cup \operatorname{Rev}_{B^{w}}^{i}$. Notice that we cannot do the same in the strong FIFO setting, since the exponential bound $B^{s}$ would lead to a co-NEXPTIME algorithm. Instead, we construct a finite automaton, whose language is empty iff the product is existentially $B^{s}$ bounded. Each state can be described in polynomial space w.r.t. $\left|G_{1}\right|,\left|G_{2}\right|$ and $\left\|B^{s}\right\|=\log _{2}\left(B^{s}\right)$ written in binary.

\section{$\star$ General outline of the co-NP-completeness reduction}

We prove the co-NP hardness of the problem of deciding either the existentialboundedness or the existential- $B$-boundedness of the product of languages of two MSC-graphs. We do not use the contents of the messages, hence the reduction holds for both weak and strong FIFO semantics. Let $\phi$ be a 3-CNF-SAT instance, with $n$ variables and $m$ clauses. This formula is true iff for each clause, one can choose a literal of the clause to be true, and no conflict occurs on a variable (one cannot choose a literal and its opposite being true). Let $B>m+1$. We build two MSC-graphs $G_{1}$ and $G_{2}$ on processes $\left\{p, q, r, p_{i}, p_{i}^{\prime} \mid 1 \leq i \leq n\right\}$ such that $G_{1} \|_{\mathrm{mp}} G_{2}$ is $\exists$ - $B$-bounded iff $\phi$ is non satisfiable. We let $m p=p$.
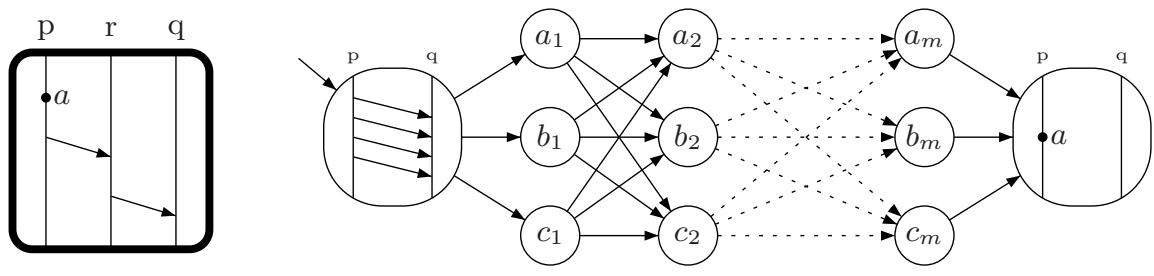

Fig. 5. MSC $M_{1}$ and MSC-graph $G_{2}$

Graph $G_{1}$ is made of one node, both initial and final. The node is labeled by MSC $M_{1}$, which is a synchronization action $a$ on process $p$, then a message from $p$ to $r$, then a message from $r$ to $q$. For graph $G_{2}$, the initial node is labeled with $B+1$ messages from $p$ to $q$. Then $G_{2}$ has a succession of $m$ choices between three nodes $a_{i}, b_{i}, c_{i}, i \leq m$. Then the final node of $G_{2}$ is labeled by the synchronization event $a$ on process $p$. Informally, the $m$ choices correspond to the $m$ clauses, and $a_{i}, b_{i}, c_{i}$ correspond to the choice of the first, second and third literal true in the $i$-th clause. That is, if the first literal in the $i$-th clause is $v_{j}$, then $a_{i}$ is labeled by a message from $q$ to $p_{j}$ and a message from $p_{j}^{\prime}$ to $p$. If the first literal in the $i$-th clause is $\neg v_{j}$, then $a_{i}$ is labeled by a message from $q$ to $p_{j}^{\prime}$ and a message from $p_{j}$ to $p$. Any MSC from $G_{2}$ corresponds to some choice of literal true in each of the clauses and vice versa. Now, a conflict occurs on one variable iff the receptions on $q$ from $p$ (in $G_{2}$ ) are before the synchronization event $a$, iff for the corresponding MSC $M_{2}$ of $G_{2}$, all MSCs in $M_{2} \|_{\mathrm{mp}} M_{1}$ are $\exists$-B-bounded. 


\section{CMSC-Graph Representation of a Monitored Product}

In the case where $\mathcal{L}\left(G_{1}\right) \|_{\mathrm{mp}} \mathcal{L}\left(G_{2}\right)$ is $\exists$-bounded, one may wish to compute a safe CMSC-graph representation of this MSC-language, which can be input to existing tools for analyzing MSC-graphs (MSCan, SOFAT...). For this purpose, we use the results from [4, where a syntax-semantics correspondence is established between globally cooperative CMSC-graphs [7, and MSC-languages $\mathcal{X}$ with regular representative sets $\mathcal{L i n}^{B}(\mathcal{X})$ for some $B>0$.

Definition 7. $G=\left(V, \rightarrow, \Lambda, V^{0}, V^{f}\right)$ is a globally cooperative $C M S C$-graph if

- $G$ is a safe $C M S C$-graph, and

- for any circuit $v_{1} \ldots v_{n}$ in $G$, all CMSCs in the set $\Lambda\left(v_{1}\right) \cdot \ldots \cdot \Lambda\left(v_{n}\right)$ have connected communication graphs.

The communication graph induced by $X=\left(E, \lambda, \mu,\left(<_{p}\right)_{p \in \mathcal{P}}\right)$ is the undirected graph $(Q, E)$ with the set of vertices $Q=\left\{p \in \mathcal{P} \mid(\exists e \in E) \quad \lambda(e) \in \mathcal{S}_{p} \cup \mathcal{R}_{p}\right\}$ and with the set of edges $E=\left\{\{p, q\} \mid\left(\exists e_{1}, e_{2} \in E\right)(\exists m \in \mathcal{M}) \lambda\left(e_{1}\right)=p ! q(m) \wedge\right.$ $\left.\lambda\left(e_{2}\right)=q ? p(m)\right\}$.

Notice that the MSC-graph from Fig. [3 is globally cooperative. Thus, boundedness of the product of $\mathcal{L}\left(G_{1}\right)$ and $\mathcal{L}\left(G_{2}\right)$ stays undecidable even when both $G_{1}, G_{2}$ are globally cooperative (Theorem 11). Quite remarkably, $\mathcal{L}\left(G_{1}\right) \cap \mathcal{L}\left(G_{2}\right)=\emptyset$ is decidable as soon as $G_{1}$ or $G_{2}$ is globally cooperative 7 .

Theorem 3. Let $\mathcal{X}$ be a set of MSCs. The following are equivalent:

- $\mathcal{X}=\mathcal{L}(G)$ for some globally cooperative CMSC-graph $G$,

$-\mathcal{L i n}^{B}(\mathcal{X})$ is a regular representative set for $\mathcal{X}$ for sufficiently large $B>0$. Moreover, $B$ and a finite automaton recognizing $\mathcal{L i n}^{B}(\mathcal{X})$ can be computed effectively from $G$. Conversely, $G$ can be computed effectively from $\operatorname{Lin}^{B}(\mathcal{X})$.

The statement of Theorem 3 is the same as (a fragment of) the main theorem of 4. However, we consider in this paper messages with contents, while [4] does not. Instead of proving Theorem 3 from scratch, we derive it from [4. The strong FIFO case comes directly from the proof of 4 . For weak FIFO, we use a translation from sets of weak FIFO MSCs to sets of FIFO MSCs with exactly one (type of) message $m$ (hence they embed in weak FIFO MSCs). In few words, the translation adds as many processes as types of messages per channel, and it preserves the existential boundedness of sets of MSCs, although the bound $B$ may grow to $3 B$. Once this translation is defined, the proof of Theorem 3 is almost immediate.

Now let $G_{1}, G_{2}$ be two globally cooperative CMSC-graphs. If $\mathcal{L}\left(G_{1}\right) \|_{\mathrm{mp}}$ $\mathcal{L}\left(G_{2}\right)$ is $\exists$-bounded, then this MSC-language is $\exists$-B-bounded, for $B \in\left\{B^{s}, B^{w}\right\}$ as defined in Prop. 4. Therefore, $\operatorname{Lin}^{B}\left(\mathcal{L}\left(G_{1}\right) \|_{\text {mp }} \mathcal{L}\left(G_{2}\right)\right)$ is a representative set for $\mathcal{L}\left(G_{1}\right) \|_{\mathrm{mp}} \mathcal{L}\left(G_{2}\right)$. By Prop. 2 $\mathcal{L}_{i n}{ }^{B}\left(\mathcal{L}\left(G_{1}\right) \|_{\mathrm{mp}} \mathcal{L}\left(G_{2}\right)\right)=\mathcal{L}_{i n}{ }^{B}\left(\mathcal{L}\left(G_{1}\right)\right) \|_{\mathrm{mp}}$ $\mathcal{L}^{B}{ }^{B}\left(\mathcal{L}\left(G_{2}\right)\right) \cap \mathcal{L} i n^{B}$. Since both $G_{1}, G_{2}$ are globally cooperative, we get that both $\mathcal{L}_{i n}{ }^{B}\left(\mathcal{L}\left(G_{1}\right)\right)$ and $\mathcal{L i n}^{B}\left(\mathcal{L}\left(G_{2}\right)\right)$ are regular and effectively computable. Since the shuffle of regular language is regular, we get the following. 
Theorem 4. Let $G_{1}, G_{2}$ be two globally cooperative CMSC-graphs such that $\mathcal{L}\left(G_{1}\right) \|_{\mathrm{mp}} \mathcal{L}\left(G_{2}\right)$ is $\exists$-bounded. Then one can effectively compute a globally cooperative CMSC-graph $G$ with $\mathcal{L}(G)=\mathcal{L}\left(G_{1}\right) \|_{\mathrm{mp}} \mathcal{L}\left(G_{2}\right)$. Moreover, $G$ is of size at most exponential and doubly exponential in the size of $\left|G_{1}\right|,\left|G_{2}\right|$, respectively with weak and strong FIFO.

\section{Conclusion}

We presented a framework to work with the controlled products of distributed components, granted that synchronizations are operated on a single monitor process, and components are given as globally cooperative CMSC-graphs. Namely, one can test whether the monitored product of components can be represented as a globally cooperative CMSC-graph. In that case, a complete analysis of the product system can be performed with existing tools. We analyze the problem in both weak and strong FIFO contexts. Weak FIFO enjoys a better complexity, while strong FIFO allows us to use non-synchronized actions with common names on different components (it suffices to rename the actions according to components, perform the product, and then rename the actions back). A direction for future work is to propose guidelines and tools for modeling product systems with one monitor process.

\section{References}

1. Alur, R., Etessami, K., Yannakakis, M.: Realizability and Verification of MSC Graphs. In: Orejas, F., Spirakis, P.G., van Leeuwen, J. (eds.) ICALP 2001. LNCS, vol. 2076, pp. 797-808. Springer, Heidelberg (2001)

2. Caillaud, B., Darondeau, P., Hélouët, L., Lesventes, G.: HMSCs as Partial Specifications.. with PNs as Completions. In: Cassez, F., Jard, C., Rozoy, B., Dermot, M. (eds.) MOVEP 2000. LNCS, vol. 2067, pp. 125-152. Springer, Heidelberg (2001)

3. Duboc, C.: Mixed Product and Asynchronous Automata. Theoretical Computer Science 48(3), 183-199 (1986)

4. Genest, B., Kuske, D., Muscholl, A.: A Kleene Theorem and Model Checking for a Class of Communicating Automata. Inf. Comput. 204(6), 920-956 (2006)

5. Gunter, E., Muscholl, A., Peled, D.: Compositional Message Sequence Charts. STTT 5(1), 78-89, (2003); In: Margaria, T., Yi, W. (eds.) TACAS 2001. LNCS, vol. 2031, pp. 496-511. Springer, Heidelberg (2001)

6. Genest, B., Muscholl, A., Peled, D.: Message Sequence Charts. In: Desel, J., Reisig, W., Rozenberg, G. (eds.) ACPN 2003. LNCS, vol. 3098, pp. 537-558. Springer, Heidelberg (2004)

7. Genest, B., Muscholl, A., Seidl, H., Zeitoun, M.: Infinite-state High-level MSCs: Model-checking and Realizability. JCSS 72(4), 617-647 (2006); Widmayer, P., Triguero, F., Morales, R., Hennessy, M., Eidenbenz, S., Conejo, R. (eds.): ICALP 2002. LNCS, vol. 2380, pp. 617-647. Springer, Heidelberg (2002)

8. Hélouët, L., Jard, C.: Conditions for synthesis of communicating automata from HMSCs. In: FMICS 2000, pp. 203-224 (2000)

9. Henriksen, J.G., Mukund, M., Kumar, K.N., Sohoni, M.A., Thiagarajan, P.S.: A theory of regular MSC languages. Inf. Comput. 202(1), 1-38 (2005) 
10. Klein, J., Caillaud, B., Hélouët, L.: Merging scenarios. In: FMICS 2004, pp. 209226 (2004)

11. Lohrey, M., Muscholl, A.: Bounded MSC communication. Inf. Comput. 189(2), 160-181 (2004)

12. Madhusudan, P., Meenakshi, B.: Beyond Message Sequence Graphs. In: Hariharan, R., Mukund, M., Vinay, V. (eds.) FSTTCS 2001. LNCS, vol. 2245, pp. 256-267. Springer, Heidelberg (2001)

13. Morin, R.: Recognizable Sets of Message Sequence Charts. In: Alt, H., Ferreira, A. (eds.) STACS 2002. LNCS, vol. 2285, pp. 523-534. Springer, Heidelberg (2002)

14. Mukund, M., Kumar, K.N., Sohoni, M.A.: Bounded time-stamping in messagepassing systems. TCS 290(1), 221-239 (2003)

15. Muscholl, A., Peled, D., Su, Z.: Deciding properties of Message Sequence Charts. In: Nivat, M. (ed.) FOSSACS 1998. LNCS, vol. 1378, pp. 226-242. Springer, Heidelberg (1998)

16. Muscholl, A., Peled, D.: Message Sequence Graphs and Decision Problems on Mazurkiewicz Traces. In: Kutyłowski, M., Wierzbicki, T., Pacholski, L. (eds.) MFCS 1999. LNCS, vol. 1672, pp. 81-91. Springer, Heidelberg (1999) 